\title{
EIGENVALUES OF THE $p$-LAPLACIAN AND DISCONJUGACY CRITERIA
}

\author{
PABLO L. DE NAPOLI AND JUAN P. PINASCO
}

Received 6 September 2005; Revised 6 March 2006; Accepted 15 March 2006

We derive oscillation and nonoscillation criteria for the one-dimensional $p$-Laplacian in terms of an eigenvalue inequality for a mixed problem. We generalize the results obtained in the linear case by Nehari and Willett, and the proof is based on a Picone-type identity.

Copyright (c) 2006 P. L. De Napoli and J. P. Pinasco. This is an open access article distributed under the Creative Commons Attribution License, which permits unrestricted use, distribution, and reproduction in any medium, provided the original work is properly cited.

\section{Introduction}

In this work we study the following equation:

$$
\left(\left|u^{\prime}\right|^{p-2} u^{\prime}\right)^{\prime}+q(t)|u|^{p-2} u=0
$$

Here, $1<p<\infty, t \in[a,+\infty)$, and $q(t)$ is a nonnegative continuous function not vanishing in subintervals of the form $(b,+\infty)$.

The solutions of (1.1) are classified as oscillatory or nonoscillatory. In the first case, a solution has an infinite number of isolated zeros; in the second case, a solution has a finite number of zeros. However, from the Sturm-Liouville theory for the $p$-laplacian $([11,16,22]$; see also the recent monograph $[10])$ if one solution is oscillatory (resp., nonoscillatory), then every solution is oscillatory (resp., nonoscillatory). Hence, we may speak about oscillatory or nonoscillatory equations, instead of solutions.

For the $p$-laplacian operator, there are several criteria for oscillation and nonoscillation in the literature; see for example [6-9]. Among the class of nonoscillatory equations, when any solution has at most one zero in $[a,+\infty)$, the equation is called disconjugate on $[a,+\infty)$.

The disconjugacy phenomenon is considerably more difficult and less understood than nonoscillation; we refer the interested reader to the surveys $[3,5,23]$ for the linear case $p=2$. 
We consider first the disconjugacy problem on $[a,+\infty)$. The relationship between disconjugacy and the eigenvalues of a mixed problem

$$
-u^{\prime \prime}=\lambda q(t) u, \quad u(a)=0=u^{\prime}(b)
$$

is due to Nehari [17], and was generalized to different equations in [14, 20, 24]. We prove here the following theorems generalizing some of their results for the $p$-laplacian.

THeORem 1.1. Let $\lambda_{1}$ be the first eigenvalue of

$$
\left(\left|u^{\prime}\right|^{p-2} u^{\prime}\right)^{\prime}+\lambda q(t)|u|^{p-2} u=0, \quad u(a)=0=u^{\prime}(b), \quad a<b,
$$

then (1.1) is disconjugate in $[a,+\infty)$ if and only if $\lambda_{1}>1$ for all $b>a$.

Also, we have the following result for oscillatory equations.

THeOREM 1.2. Equation (1.1) is oscillatory if and only if there exists a sequence of intervals $\left[a_{n}, b_{n}\right]$ with $a_{n}<b_{n}, a_{n} \nearrow+\infty$ as $n \nearrow+\infty$ such that the first eigenvalue $\lambda_{1}^{(n)}$ of

$$
\left(\left|u^{\prime}\right|^{p-2} u^{\prime}\right)^{\prime}+\lambda q(t)|u|^{p-2} u=0, \quad u\left(a_{n}\right)=0=u^{\prime}\left(b_{n}\right)
$$

satisfies $\lambda_{1}^{(n)} \leq 1$ for $n \geq 1$.

For the linear case $p=2$ and more general functions $q(t)$, the proof in [23] follows by analyzing a Lagrange identity formed by a positive solution of (1.1) and an eigenfunction, and by using Riccati equation techniques. Our main tool for the proof of both theorems is a Picone type identity as in $[1,13]$ and the variational characterization of the first eigenvalue which can be obtained from the Rayleigh quotient (see [4, 12], and also [19] for the equivalence between several abstract formulations),

$$
\lambda_{1}=\inf _{u \in W} \frac{\int_{a}^{b}\left|u^{\prime}\right|^{p} d t}{\int_{a}^{b} q(t)|u|^{p} d t},
$$

where $W=W_{0}^{1, p}(a, b) \backslash\{0\}$ for the Dirichlet boundary condition, and $W=\left\{u \in W^{1, p}(a\right.$, b) $: u(a)=0\} \backslash\{0\}$ for the mixed problem (1.3).

As an application, we prove Leighton-Wintner theorem (see $[7,8])$ for oscillation in the half line.

Let us note that the eigenvalue problem for the $p$-laplacian has been widely studied in recent years; see, for example, $[2,22]$ among several others, and the references therein. Hence, a characterization for disconjugacy in terms of eigenvalues could be a useful tool.

Moreover, we also consider the disconjugacy phenomenon in a bounded closed interval $[a, b]$. We will show that the existence of two zeros in $[a, b]$ of a solution $u$ is related with the Dirichlet eigenvalue problem

$$
-\left(\left|u^{\prime}\right|^{p-2} u^{\prime}\right)^{\prime}=\lambda q(t)|u|^{p-2} u, \quad u(a)=0=u(b),
$$

and (1.1) is disconjugate if and only if the first eigenvalue satisfies $\lambda_{1}>1$. One implication follows from the Sturmian comparison theorem. 
Lemma 1.3. If $(1.1)$ is disconjugate on $[a, b]$, then the first Dirichlet eigenvalue is greater than one.

In order to prove the other implication, it is convenient to introduce the $p$-degree functional

$$
F(u ; a, b)=\int_{a}^{b}\left|u^{\prime}\right|^{p}-q(t)|u|^{p} d t
$$

which is positive for every $u \in W_{0}^{1, p}(a, b)$ not identically zero on $(a, b)$ if and only if $(1.1)$ is disconjugate on $[a, b]$. This equivalence can be found in the so-called Roundabout theorem [8]. Hence, we have another equivalent criterion for disconjugacy on bounded closed intervals.

As far as we know, this fact was not observed previously even for the linear case. Therefore, the problem of finding disconjugacy conditions on finite intervals is related to the problem of find lower bounds for eigenvalues. The search of lower bounds of eigenvalues has a long history, which can be traced back to Sturm and Liouville; lower bounds for the p-Laplacian eigenvalues were obtained in [18] by generalizing Lyapunov inequality [15], a classical tool in oscillation theory.

The paper is organized as follows: in Section 2 we prove the main Theorems 1.1 and 1.2; in Section 3 we prove Lemma 1.3 and discuss its relationship with the Roundabout theorem.

\section{Main theorems}

Our main tool is the following Picone-type identity which can be found in [1].

Theorem 2.1. Let $v>0, u \geq 0$ be differentiable a.e. in $(a, b)$. Denote

$$
\begin{gathered}
L(u, v)=\left|u^{\prime}\right|^{p}+(p-1) \frac{u^{p}}{v^{p}}\left|v^{\prime}\right|^{p}-p \frac{u^{p-1}}{v^{p-1}}\left|v^{\prime}\right|^{p-2} v^{\prime} u^{\prime} \\
R(u, v)=\left|u^{\prime}\right|^{p}-\left|v^{\prime}\right|^{p-2} v^{\prime}\left(\frac{u^{p}}{v^{p-1}}\right)^{\prime} .
\end{gathered}
$$

Then,

(i) $L(u, v)=R(u, v)$,

(ii) $L(u, v) \geq 0$ a.e. in $(a, b)$,

(iii) $L(u, v)=0$ a.e. in $(a, b)$ if and only if $u=k v$ for some $k \in \mathbb{R}$.

We are ready to prove Theorems 1.1 and 1.2.

Proof of Theorem 1.1. Let us assume that (1.1) is disconjugate, and let us prove that $\lambda_{1}>1$. To this end we suppose that $\lambda_{1} \leq 1$ and first we will show that $\lambda_{1}<1$ is not possible in any interval $[a, b]$. Then, if $\lambda_{1}=1$ for some interval, we will find a larger interval $[a, c]$ such that the corresponding first eigenvalue satisfies $\lambda_{1}^{(c)}<1$, a contradiction.

Hence, we suppose that there exist $b>a$ and $\lambda \leq 1$ such that problem (1.3) has a nontrivial eigenfunction $u$. Also, since (1.1) is disconjugate, there exists a positive solution $v$ of $(1.1)$ on $[a,+\infty)$. Then, $v^{\prime}(t)>0$ on $[a,+\infty)$. 
From the definition of $R(u, v)$ in Theorem 2.1, we have

$$
\int_{a}^{b} R(u, v) d t=\int_{a}^{b}\left|u^{\prime}\right|^{p} d t-\int_{a}^{b}\left|v^{\prime}\right|^{p-2} v^{\prime}\left(\frac{u^{p}}{v^{p-1}}\right)^{\prime} d t
$$

Now, we use the weak formulation of Problems (1.1) and (1.3), multiplying the first by $\left(u^{P} / v^{p-1}\right)$ and the second by $u$, obtaining

$$
\int_{a}^{b} R(u, v) d t+\left|v^{\prime}(b)\right|^{p-2} v^{\prime}(b) \frac{u^{p}(b)}{v^{p-1}(b)}=(\lambda-1) \int_{a}^{b} q(t)|u|^{p} d t .
$$

Since $R(u, v)=L(u, v) \geq 0$, and we assume that $\lambda \leq 1$, we have $\lambda=1$.

Let us take $c>b$, and let us consider the eigenvalue problem in $(a, c)$ :

$$
\left(\left|w^{\prime}\right| p^{p-2} w^{\prime}\right)^{\prime}+\lambda q(t)|w|^{p-2} w=0, \quad w(a)=0=w^{\prime}(c) .
$$

We extend the eigenfunction $u$ as $u(b)$ in $(b, c)$, and let us call it $\tilde{u}$. Since $\tilde{u}$ is an admissible function in the variational characterization of the first eigenvalue $\lambda_{1}^{(c)}$ in $(a, c)$, we obtain

$$
\lambda_{1}^{(c)}=\inf _{w \in W^{1, p}(a, c), w(a)=0} \frac{\int_{a}^{c}\left|w^{\prime}\right|^{p} d t}{\int_{a}^{c} q(t)|w|^{p} d t} \leq \frac{\int_{a}^{c}\left|\tilde{u}^{\prime}\right|^{p} d t}{\int_{a}^{c} q(t)|\tilde{u}|^{p} d t}=\frac{\int_{a}^{b}\left|u^{\prime}\right|^{p} d t}{\int_{a}^{b} q(t)|u|^{p} d t+\int_{b}^{c} q(t) u^{p}(b) d t} .
$$

Let us observe that $\lambda_{1}^{(c)}<1$ unless $q(t) \equiv 0$ in $(b, c)$. Since this argument is valid for each $c>b$, and $q(t)$ cannot vanish identically on intervals of the form $(b,+\infty)$, there exists an interval $(a, c)$ where the first eigenvalue satisfies $\lambda_{1}^{(c)}<1$, which is not possible by the previous argument.

Let us prove now the converse. Let us assume that the first eigenvalue $\lambda_{1}$ of

$$
\left(\left.\left|u^{\prime}\right|\right|^{p-2} u^{\prime}\right)^{\prime}+\lambda q(t)|u|^{p-2} u=0, \quad u(a)=0=u^{\prime}(b), \quad a<b
$$

is greater than 1 . If (1.1) is not disconjugate in $[a,+\infty)$, there exists a solution $v$ with two zeros $t_{1}, t_{2} \in[a,+\infty)$. Now, we choose $v$ as a test function for the eigenvalue problem in $\left[a, t_{2}\right]$, extending it by zero in $\left[a, t_{1}\right)$. Clearly, the Rayleigh quotient gives

$$
\lambda_{1}=\inf _{u \in W^{1, p}(a, b), u(a)=0} \frac{\int_{a}^{b}\left|u^{\prime}\right|^{p} d t}{\int_{a}^{b} q(t)|u|^{p} d t} \leq \frac{\int_{t_{1}}^{t_{2}}\left|v^{\prime}\right|^{p} d t}{\int_{t_{1}}^{t_{2}} q(t)|v|^{p} d t}=1,
$$

a contradiction. Hence, (1.1) must be disconjugate.

Proof of Theorem 1.2. Let us assume first that (1.1) is oscillatory. Therefore, there exists a solution $v$ with infinitely many zeros $a<t_{1}<t_{2}<\ldots \nearrow+\infty$. Let us choose $a_{n}=t_{n}, b_{n}=$ $t_{n+1}$. The first Dirichlet eigenfunction in $\left[a_{n}, b_{n}\right]$ coincides with $v$ up to a multiplicative constant, with eigenvalue equal to 1 . The eigenvalue $\lambda_{1}^{(n)}$ of

$$
\left(\left|u^{\prime}\right| p^{p-2} u^{\prime}\right)^{\prime}+\lambda q(t)|u|^{p-2} u=0, \quad u\left(a_{n}\right)=0=u^{\prime}\left(b_{n}\right)
$$


satisfies $\lambda_{1}^{(n)} \leq 1$, since $v$ is an admissible function in the variational characterization of it, and

$$
\lambda_{1}^{(n)}=\inf _{u \in W^{1, p}\left(a_{n}, b_{n}\right), u\left(a_{n}\right)=0} \frac{\int_{a_{n}}^{b_{n}}\left|u^{\prime}\right|^{p} d t}{\int_{a_{n}}^{b_{n}} q(t)|u|^{p} d t} \leq \frac{\int_{a_{n}}^{b_{n}}\left|v^{\prime}\right|^{p} d t}{\int_{a_{n}}^{b_{n}} q(t)|v|^{p} d t}=1 .
$$

Suppose now that the eigenvalue condition is satisfied for a family of intervals $\left[a_{n}, b_{n}\right]$. Let us suppose that there exists a nonoscillatory solution $u$, and let us take one of the intervals with $a_{N}$ greater than the last zero of $u$. Therefore, $(1.1)$ is disconjugate in $\left[a_{N},+\infty\right)$ (if not, there exists a solution with two zeros, and the Sturmian theory implies that $u$ must have a zero between them). Hence, from Theorem 1.1 we get $\lambda_{1}^{(N)}>1$, a contradiction.

From Theorem 1.2 we have the following classical oscillation result.

Theorem 2.2 (Leighton-Wintner theorem). If $\int_{a}^{+\infty} q(t) d t=+\infty$, then (1.1) is oscillatory on $[a,+\infty)$.

Proof. The proof follows from Theorem 1.2. For any $a_{n} \geq a$, we choose $b_{n}$ such that

$$
\int_{a_{n}+1}^{b_{n}} q(t) d t \geq 1
$$

and we compute the Rayleigh quotient for the first eigenvalue $\lambda_{1}^{(n)}$ of the mixed problem

$$
\left(\left|u^{\prime}\right|^{p-2} u^{\prime}\right)^{\prime}+\lambda q(t)|u|^{p-2} u=0, \quad u\left(a_{n}\right)=0=u^{\prime}\left(b_{n}\right)
$$

with the test function

$$
v= \begin{cases}t-a_{n} & \text { if } t \in\left[a_{n}, a_{n}+1\right) \\ 1 & \text { if } t \in\left[a_{n}+1, b_{n}\right] .\end{cases}
$$

Hence,

$$
\lambda_{1}^{(n)}=\inf _{u \in W^{1, p}\left(a_{n}, b_{n}\right), u\left(a_{n}\right)=0} \frac{\int_{a_{n}}^{b_{n}}\left|u^{\prime}\right|^{p} d t}{\int_{a_{n}}^{b_{n}} q(t)|u|^{p} d t} \leq \frac{\int_{a_{n}}^{b_{n}}\left|v^{\prime}\right|^{p} d t}{\int_{a_{n}}^{b_{n}} q(t)|v|^{p} d t}<1 .
$$

Remark 2.3. A different proof of this theorem can be found in [10], without sign condition on $q(t)$.

\section{Disconjugacy on bounded intervals}

In this section we consider the disconjugacy problem on a bounded closed interval. We prove first Lemma 1.3.

Proof of Lemma 1.3. Let us suppose that the first eigenvalue $\lambda_{1}$ of

$$
\left(\left|u^{\prime}\right|^{p-2} u^{\prime}\right)^{\prime}+\lambda q(t)|u|^{p-2} u=0, \quad u(a)=0=u(b)
$$

satisfies $\lambda_{1} \leq 1$. 
If $\lambda_{1}=1$, the corresponding eigenfunction $u$ satisfies $u(a)=0=u(b)$. Therefore (1.1) is not disconjugate.

If $\lambda_{1}<1$, let us consider the unique solution $u$ of

$$
\left(\left|u^{\prime}\right| p^{p-2} u^{\prime}\right)^{\prime}+q(t)|u|^{p-2} u=0
$$

satisfying

$$
u(a)=0, \quad u^{\prime}(a)=1 .
$$

From the Sturmian oscillation theory, we conclude that $u$ has a zero $c$ between $a$ and $b$, and (1.1) is not disconjugate.

Now we state the Roundabout theorem. Lemma 1.3 proves $(\mathrm{i}) \Rightarrow(\mathrm{v})$; we will prove only (v) $\Rightarrow$ (iv).

Theorem 3.1 (Roundabout theorem). Let $q(t) \geq 0, q(t) \neq 0$ on $[a, b]$. The following statements are equivalent.

(i) Equation (1.1) is disconjugate on an interval $I=[a, b]$; that is, any nontrivial solution of (1.1) has at most one zero in I.

(ii) There exists a solution of (1.1) having no zero in $[a, b]$.

(iii) There exists a solution $w$ of the generalized Riccati equation corresponding to (1.1),

$$
w^{\prime}+q(t)+(p-1)|w|^{p^{\prime}}=0, \quad p^{\prime}=\frac{p}{p-1},
$$

which is defined on the whole interval $[a, b]$.

(iv) The p-degree functional

$$
F(u ; a, b)=\int_{a}^{b}\left|u^{\prime}\right|^{p}-q(t)|u|^{p} d t
$$

is positive for every $u \in W_{0}^{1, p}(a, b)$, u not identically zero on $I$.

(v) The first eigenvalue $\lambda_{1}$ of

$$
\left(\left|u^{\prime}\right|^{p-2} u^{\prime}\right)^{\prime}+\lambda q(t)|u|^{p-2} u=0, \quad u(a)=0=u(b)
$$

satisfies $\lambda_{1}>1$.

Proof. From the variational characterization of the first eigenvalue,

$$
\lambda_{1}=\inf _{u \in W_{0}^{1, p}(a, b)} \frac{\int_{a}^{b}\left|u^{\prime}\right| p d t}{\int_{a}^{b} q(t)|u|^{p} d t} .
$$

Hence, if $\lambda_{1}>1$, we have that

$$
\int_{a}^{b}\left|u^{\prime}\right|^{p} d t-\int_{a}^{b} q(t)|u|^{p} d t \geq\left(\lambda_{1}-1\right) \int_{a}^{b} q(t)|u|^{p} d t>0
$$

for every $u \in W_{0}^{1, p}(a, b), u \neq 0$. 
Remark 3.2. The eigenvalue problem in unbounded intervals was studied in [21]. However, it is not known if the eigenvalues can be characterized variationally.

\section{Acknowledgments}

We want to thank the referees for several comments improving the manuscript. The first author is supported by Fundacion Antorchas and CONICET. The second author is supported by Fundacion Antorchas and ANPCyT under Grant PICT 03-05009.

\section{References}

[1] W. Allegretto and Y. X. Huang, A Picone's identity for the p-Laplacian and applications, Nonlinear Analysis. Theory, Methods \& Applications 32 (1998), no. 7, 819-830.

[2] A. Anane, O. Chakrone, and M. Moussa, Spectrum of one dimensional p-Laplacian operator with indefinite weight, Electronic Journal of Qualitative Theory of Differential Equations 2002 (2002), no. $17,1-11$.

[3] J. H. Barrett, Oscillation theory of ordinary linear differential equations, Advances in Mathematics 3 (1969), no. 4, 415-509.

[4] F. E. Browder, Infinite dimensional manifolds and non-linear elliptic eigenvalue problems, Annals of Mathematics. Second Series 82 (1965), no. 3, 459-477.

[5] W. A. Coppel, Disconjugacy, Lecture Notes in Mathematics, vol. 220, Springer, Berlin, 1971.

[6] M. del Pino, M. Elgueta, and R. Manásevich, Generalizing Hartman's oscillation result for $\left(\left|x^{\prime}\right| p^{p-2} x^{\prime}\right)^{\prime}+c(t)|x|^{p-2} x=0, p>1$, Houston Journal of Mathematics 17 (1991), no. 1, 63-70.

[7] M. del Pino and R. Manásevich, Oscillation and nonoscillation for $\left(\left|u^{\prime}\right|^{p-2} u^{\prime}\right)^{\prime}+a(t)|u|^{p-2} u=$ 0, $p>1$, Houston Journal of Mathematics 14 (1988), no. 2, 173-177.

[8] O. Došlý, Qualitative theory of half-linear second order differential equations, Mathematica Bohemica 127 (2002), no. 2, 181-195.

[9] O. Došlý and S. Peña, A linearization method in oscillation theory of half-linear second-order differential equations, Journal of Inequalities and Applications 2005 (2005), no. 5, 535-545.

[10] O. Došlý and P. Řehák, Half-Linear Differential Equations, Elsevier, Amsterdam, 2005.

[11] Á. Elbert, Oscillation and nonoscillation theorems for some nonlinear ordinary differential equations, Ordinary and Partial Differential Equations (Dundee, 1982), Lecture Notes in Math., vol. 964, Springer, Berlin, 1982, pp. 187-212.

[12] S. Fučík and J. Nečas, Ljusternik-Schnirelmann theorem and nonlinear eigenvalue problems, Mathematische Nachrichten 53 (1972), 277-289.

[13] J. Jaroš, K. Takaŝi, and N. Yoshida, Picone-type inequalities for nonlinear elliptic equations and their applications, Journal of Inequalities and Applications 6 (2001), no. 4, 387-404.

[14] W. Kim, Disconjugacy and comparison theorems for second order linear systems, SIAM Journal on Mathematical Analysis 354 (2002), 4751-4767.

[15] A. Liapounov, Problème Général de la Stabilité du Mouvement, Annals of Mathematics Studies, vol. 17, Princeton University Press, New Jersey, 1949, reprinted from Annales de la Faculté des Sciences de Toulouse 9 (1907), 203-474, translation of original paper published in Communications of the Society of Mathematics, Kharkow (1892).

[16] J. D. Mirzov, On some analogs of Sturm's and Kneser's theorems for nonlinear systems, Journal of Mathematical Analysis and Applications 53 (1976), no. 2, 418-425.

[17] Z. Nehari, Oscillation criteria for second-order linear differential equations, Transactions of the American Mathematical Society 85 (1957), no. 2, 428-445. 
[18] J. P. Pinasco, Lower bounds for eigenvalues of the one-dimensional p-Laplacian, Abstract and Applied Analysis 2004 (2004), no. 2, 147-153.

[19] R. C. Riddell, Nonlinear eigenvalue problems and spherical fibrations of Banach spaces, Journal of Functional Analysis 18 (1975), no. 3, 213-270.

[20] K. D. Shere, Nonoscillation of second-order, linear differential equations with retarded argument, Journal of Mathematical Analysis and Applications 41 (1973), no. 2, 293-299.

[21] K. Takaŝi and M. Naito, On the number of zeros of nonoscillatory solutions to half-linear ordinary differential equations involving a parameter, Transactions of the American Mathematical Society 354 (2002), no. 12, 4751-4767.

[22] W. Walter, Sturm-Liouville theory for the radial $\Delta_{p}$-operator, Mathematische Zeitschrift 227 (1998), no. 1, 175-185.

[23] D. Willett, Classification of second order linear differential equations with respect to oscillation, Advances in Mathematics 3 (1969), no. 4, 594-623.

[24] N. Yoshida, Nonoscillation of elliptic differential equations of second order, Hiroshima Mathematical Journal 4 (1974), 279-284.

Pablo L. De Napoli: Departamento de Matematica, FCEyN, Universidad de Buenos Aires,

Ciudad Universitaria, Pabellon I, 1428 Buenos Aires, Argentina

E-mail address:pdenapo@dm.uba.ar

Juan P. Pinasco: Instituto de Ciencias, Universidad Nacional de General Sarmiento,

J.M. Gutierrez 1150, Los Polvorines, 1613 Buenos Aires, Argentina

E-mail addresses: jpinasco@ungs.edu.ar; jpinasco@dm.uba.ar 